\title{
Validación de la escala de violencia obstétrica y pruebas de la invarianza factorial en una muestra de mujeres chilenas
}

\section{Validation and factorial invariance test of obstetric violence scale \\ in women from Chile}

\author{
Manuel Cárdenas Castro ${ }^{1}$ y Stella Salinero Rates ${ }^{2}$
}

\begin{abstract}
${ }^{1}$ Psicólogo. Doctor en Comportamiento Social y Organizacional (Universidad Autónoma de Madrid). Investigador de la Facultad de Psicología de la Universidad de Talca, Chile. Investigador titular del Proyecto Anillos SOC180007 (PIA/ANID) e Investigador Responsable del Proyecto Fondecyt Regular $N^{\circ} 1210102$ (Experiencias de violencia y abuso en la atención ginecológica, estrategias de protección de las mujeres y su impacto en la percepción de sí mismas, sus cuerpos y su sexualidad).E-mail: jose.cardenas@utalca.cl

${ }^{2}$ Colectiva contra la Violencia Ginecológica y Obstétrica. Licenciada en Teoría e Historia del Arte. Máster en Estudios Avanzados en Historia del Arte. Doctoranda en Estudios Interdisciplinarios sobre Pensamiento, Cultura y Sociedad de la Universidad de Valparaíso, Chile. E-mail: stellasalinero@gmail.com
\end{abstract}

Proyecto Fondecyt Regular N. ${ }^{\circ} 1210102$ de la Agencia Nacional de Investigación y Desarrollo de Chile (ANID)

Facultad de Psicología, Universidad de Talca.

Región del Maule, Chile.

\section{Resumen}

La violencia obstétrica (VO) es un problema de enorme relevancia social en nuestro contexto, ya que puede afectar psíquica y físicamente a las mujeres, al mismo tiempo que puede socavar la confianza en el sistema de salud y limitar el acceso oportuno a este por parte de las mujeres. La violencia obstétrica se ha definido como la apropiación del cuerpo de la mujer por parte del personal de salud a través de prácticas que redundan en la medicalización y patologización de sus procesos reproductivos. Esta violencia puede ser psicológica (p. e., regaños, negativa a responder a preguntas o realizar un plan de parto ajustado a sus expectativas), física (p. e., uso innecesario de la fuerza o querer causar dolor intencionalmente) y/o sexual (p. e. comentarios de natu- raleza sexual referidos a su cuerpo o genitales, etc.). También se puede verificar mediante la realización de prácticas que están desaconsejadas o estrictamente prohibidas (p. e., monitorización fetal continua, uso de oxitocina sintética para agilizar el procedimiento, negación de la libertad de movimiento, maniobra de Kristeller, episiotomía de rutina, entre otros). No existen a la fecha escalas validadas que permitan detectar violencia obstétrica en la atención de salud. Método: Se analiza la fiabilidad (alfa y omega) y se aportan pruebas de la validez (análisis factorial confirmatorio) de la escala de VO. Las participantes de la muestra fueron 367 mujeres, mayores de edad, de la región de Valparaíso (Chile), que habían tenido alguna vez un parto. Los resultados apoyan la validez de la escala de VO y la estructura unifactorial propuesta muestra 
un buen ajuste $(\mathrm{CFI}=.94, \mathrm{NFI}=.91, \mathrm{IFI}=$ .94 y RSMEA = .067); también apoyan la idea de invarianza a través de diferentes muestras $(\triangle \mathrm{CFI}<.01$ y $\triangle \mathrm{RSMEA}<.01)$, toda vez que la escala de VO demuestra ser un instrumento confiable $(\alpha=.83$ y $\omega=.88)$. Por lo tanto se concluye que la escala de VO es un instrumento fiable y válido para la detección de este tipo específico de violencia contra las mujeres. Palabras clave: escala de violencia obstétrica, continuo de violencia contra las mujeres, confiabilidad, validez, invarianza.

\section{Abstract}

Obstetric violence is a long-standing problem of great social relevance in our context, since it can affect women psychically and physically. Similarly, obstetric violence can strongly undermine confidence in the health system and limit timely access to it by women (who could avoid conducting routine examinations associated with their pregnancy and which would explain the growing proportion women who wish to deliver at home and outside the medical system). Obstetric violence (also commonly referred to as "disrespect and abuse" during childbirth and "mistreatment" during childbirth care) has been defined as the appropriation of women's bodies by health personnel through practices that become the medicalization and pathologization of their reproductive processes. This violence can be psychological (e.g. abuse, scolding, refusal to answer your questions or carry out a birth plan adjusted to your expectations), physical (e.g. use of force or intentionally wanting to cause pain) and / or sexual (e.g. tacts of a sexual nature, comments of a sexual nature referring to your body or genitals, etc.), which can also be verified in the performance of a series of practices that are discouraged or strictly prohibited (for example, continuous fetal monitoring, use of synthetic oxytocin to speed up the procedure, impaired free movement, Kristeller's maneuver, routine episiotomy, among others).
To date, there are no validated scales in our context that allow detecting obstetric violence $(\mathrm{OV})$ in health care. These forms of violence can be a traumatic experience for many women. The scale has been created having as its main reference the so-called "obstetric violence test" prepared by the association $E l$ parto es nuestro, which includes the most recurrent situations of lack of respect and abuse in obstetric care. Reliability (alpha and omega) is analyzed and validity tests (confirmatory factor analysis) of the VO scale are provided. The invariance tests aim to ensure that the construct being evaluated (in this case obstetric violence) has the same meaning for women who provide care in the private and public health systems. The participants in the sample were 367 women, all of legal age, from the Valparaíso region (Chile) and who had had a child birth at some point in their lives. These women had their deliveries in both the public and private health systems. Of the total number of women who participated in the study, $58.9 \%$ declared having experienced some situation of violence during their delivery. The results support the validity of the VO scale, the proposed unifactorial structure shows a good fit $(\mathrm{CFI}=.94, \mathrm{NFI}=.91, \mathrm{IFI}=$ .94 and RSMEA $=.067$ ). The results support the idea of invariance through the samples of women who attended their delivery in the private and public health system $(\triangle \mathrm{CFI}<$ .01 and $\triangle \mathrm{RSMEA}<.01)$, since the VO scale proves to be a reliable instrument $(\alpha=.83$ and $\omega=.88$ ) and with high levels of internal consistency (either based on total item correlations or factor loadings, which would be the most appropriate method for scales that use ordinal level of measurement). In conclusions, the VO scale is a reliable and valid instrument for the detection of this specific type of violence against women that can contribute to studies that detect abusive practices within the health system. Many of the women who do not report having experienced obstetric violence, do report the presence of some practices that are discouraged or prohibited: genital 
shaving $(66.2 \%)$, enema $(45.5 \%)$, prohibition of consuming food or water $(51.7 \%)$, indication of lying down during labor $(24.5$ $\%$ ), induction of labor using medication (36.1 $\%$ ), repeated vaginal examinations $(24.3 \%)$, compression of the abdomen (34.5\%), episiotomy $(33.6 \%)$, cesarean section $(54.1 \%)$ ) and uterine scraping without anesthesia (2 $\%$ ). The previous results indicate the normalization of violent practices and their routine performance in the framework of delivery care in public and private health services, which could make us underestimate their prevalence. Keywords: obstetric violence, disrespect and abuse during childbirth, continuous of violence against women, reliability, validity, invariance.

\section{Introducción}

El concepto de violencia obstétrica (VO) emerge como una respuesta al sistema de asistencia del parto predominante en la cultura occidental (Bellón, 2015) y se ha definido como la apropiación del cuerpo de las mujeres por parte del personal de salud mediante prácticas que devienen en la medicalización y patologización de sus procesos reproductivos (Arguedas, 2014; Belli, 2013; Bellón, 2015; Calafell, 2015; Cohen, 2016; Gonçalves da Silva et al., 2014; Infante y Leiva, 2016; Sadler et al., 2016; Tamayo, Restrepo y González, 2015), que redundan en la pérdida de autonomía de las mujeres y en un descenso significativo de su calidad de vida. La medicalización del cuerpo refiere a la excesiva intervención médica durante la gestación, parto y puerperio (Almonte, 2016; Contreras y Fernández, 2015 y Sena, 2016). Dicha patologización se ha entendido como un efecto que convierte el cuerpo materno y el parto-nacimiento en una enfermedad que conlleva un potencial riesgo (Hernández-Garré y Echeverría-Pérez, 2016; Nacer en libertad, 2015; Salinero, 2017).

La VO se traduce en prácticas concretas como la episiotomía de rutina, la maniobra de Kristeller, la cesárea sin justificación, la rotura de la membrana, el monitoreo fetal constante, el uso de oxitocina sintética para producir y acelerar las contracciones uterinas, los tactos reiterados, entre otras. Del mismo modo, puede traducirse en prácticas con efectos en el nivel psicológico, tales como amenazas, retos, infantilización, burlas $\mathrm{y}$ groserías, como también mediante la negativa de atención (Fernández-Guillén, 2015; Instituto Nacional de Derechos Humanos [INDH], 2016; INSGENAR, 2003; Majety y Bejugam, 2017; OMS, 2014).

Existe un relativo acuerdo en diferenciar la VO de otras prácticas de violencia médica, en el sentido de que estaría dirigida hacia las mujeres, en particular hacia las gestantes, parturientas y puérperas, lo cual constituye una forma específica de violencia de género arraigada en las concepciones socioculturales sobre las mujeres y los cuerpos femeninos, y que sería una expresión más del continuo de violencia hacia las mujeres (Arguedas, 2014; Belli, 2013; Calafell, 2015; Cohen, 2016; Contreras y Fernández-Guillén, 2015; Fernández y Contreras, 2015; Majety y Bejugam, 2017, Red chilena contra la violencia hacia las mujeres, 2015; Sadler et al., 2016). Se trataría de una expansión de la violencia que viven en los distintos ámbitos de sus vidas quienes son asignadas al nacer como mujeres hacia el campo de su salud sexual y reproductiva.

A la fecha no existen instrumentos apropiadamente validados para su uso en español que puedan pesquisar la magnitud de la falta de respeto y el abuso en el ámbito obstétrico por parte de los equipos de salud, aunque estas prácticas parecen estar mucho más extendidas de lo que se podría suponer (Observatorio de Violencia Obstétrica, 2018). Una serie de estudios internacionales han incluido preguntas tendientes a pesquisar el grado en que las mujeres perciben que han sido maltratadas por proveedores de servicios médicos en relación con sus características físicas y raza, edad, dominio del idioma, entre otras, en el 
marco de su atención de gestación y parto (De Marco et al., 2008). Las preguntas se realizaron en formato de respuesta dicotómico ( $S_{1}^{\prime}$ o No). Del mismo modo, otros estudios han utilizado preguntas, en el mismo formato de respuesta dicotómica, para detectar presencia o ausencia de una serie de prácticas consideradas como violentas en el marco de la atención del parto (Asefa y Bekele, 2015; Faneite et al., 2012; Kruk et al., 2014; Terán et al., 2013; Valdez-Santiago, 2015); los resultados fueron presentados simplemente en términos porcentuales y de frecuencia absoluta. Uno de los instrumentos más utilizados a la fecha es el cuestionario de abuso Norvold (Lukasse et al., 2015; Swahnberg y Wijma, 2003) que incluye tres preguntas descriptivas sobre experiencias de abuso en los cuidados de salud y que tiene una pregunta concreta sobre la experiencia en el parto. En términos generales, dichos estudios informan sobre la prevalencia de abusos en el ámbito de los cuidados de salud de 20 $\%$ para mujeres y de $8 \%$ en hombres (Brüggemann y Swahnberg, 2014), lo que pondría de manifiesto diferencias percibidas en el trato por ambos grupos. Otros estudios, de tipo cualitativo, indicaron que las mujeres definían su experiencia como sintiéndose anuladas (Swahnberg, Thapar-Björkert y Bertero, 2007). Del mismo modo, otros estudios informan que entre un 13 y un $28 \%$ de las mujeres han experimentado abuso dentro del sistema de salud en el marco de su atención ginecológica (Schroll, Kjaergaard y Midtgaard, 2013) y que dicho abuso se asociaría con morbilidad y mortalidad materna en el parto (Bowsery Hill, 2010; D'Oliveira, Diniz y Schraiber, 2002) enfatizado que la falta de respeto y el abuso en el parto constituyen causas importantes de sufrimiento y violación de los derechos humanos para las mujeres en muchos países (Respectful Maternity Care Advisory Council, 2011).

Los estudios anteriores indagan desde el uso de metodologías cuantitativas en el fenó- meno de la VO (también denominado comúnmente como "falta de respeto y abuso durante el parto" y "maltrato durante la atención del parto"), aunque no aportan información sobre el uso de algún tipo de instrumento de medición fiable y debidamente validado (Savagey Castro, 2017). De lo que sí existen pruebas suficientes es del profundo impacto que dicha violencia tiene en la salud física y psíquica de las mujeres (Salinero, 2021), ya que muchas de las prácticas que se consideran VO redundan en incisiones y cortes en el cuerpo que alteran la fisonomía del cuerpo, dejando marcas y cicatrices, que pueden derivar en relaciones sexuales dolorosas, incontinencia urinaria y problemas de autoestima, entre otros (Fernández-Guillén, 2015). Para muchas de las mujeres, la atención ginecológica puede llegar a constituir una experiencia traumática, ya que en ella se establece una relación asimétrica y donde las retóricas del cuidado (Salinero, 2017) hacen que el daño infringido por quienes se suponen que deberían cuidarlas pueda llegar a tener un efecto más profundo. Se trata de un tipo de trauma relacional (Benjamin, 2012) que opera por la falta de reconocimiento y la invisibilización de las mujeres.

El objetivo de este estudio es construir y validar un instrumento que permita pesquisar las experiencias de VO y relacionarlas, en futuros trabajos, con una serie de resultados desfavorables que van en desmedro de la salud de las mujeres y que pueden afectar también a sus hijos e hijas. Establecer una relación entre esta forma específica de violencia y los malos resultados de salud puede abrir una discusión respecto de la forma que los servicios de obstetricia deben tomar para adaptarse a las necesidades de las mujeres y contribuir a vigilar el adecuado cumplimiento de los protocolos de atención establecidos. A la fecha no existen instrumentos validados sobre abuso y violencia en la atención de salud, aun cuando se requieren con urgencia. 


\section{Método}

\section{Participantes}

El estudio se desarrolló en una muestra de carácter no probabilístico (de conveniencia) en la que participaron 367 mujeres, cuyas edades fluctuaron entre 19 y 85 años $(M=45.29$ y $D T$ $=14.19)$ residentes de la región de Valparaíso (Chile). El 96.7\% de ellas se identifica como heterosexual, un $1.7 \%$ como bisexual y un 1.7 $\%$ se identifica con otras categorías (lesbiana, fluida o no binaria). La edad de su primera consulta ginecológica fue entre los 11 y los 36 años $(M=18.32$ y $D T=4.66)$. El $69.3 \%$ de las mujeres que participó del estudio ha tenido un parto en servicios públicos de salud, mientras que un $42.5 \%$ ha tenido algún parto en clínicas privadas. Solo un $2.3 \%$ ha tenido un parto en casa. El $99.4 \%$ de ellas tuvo un parto que terminó en el nacimiento de una hija o hijo. El promedio de hijos de las mujeres de la muestra fue de 2.22 (hay mujeres que, a pesar de haber tenido un parto, no tienen hijos y mujeres que han tenido hasta siete hijos). Los cuestionarios fueron aplicados por personal especialmente entrenado por el equipo de investigadores para el estudio. Las participantes, todas voluntarias, rellenaron el cuestionario en sus casas. La administración fue individual, con una duración aproximada de entre 15 y 20 minutos. Antes de ser encuestadas, a las participantes se les informó cuáles eran los objetivos del estudio y firmaron una carta de consentimiento informado, en la que aceptaban participar en el estudio y se les garantizaba confidencialidad y anonimato. Para la selección de la muestra se definió un perfil para las encuestadas. Los criterios de selección para las participantes fueron: (1) vivir en la región en la que se desarrolló el estudio (Valparaíso); (2) haber tenido un parto alguna vez en su vida; y (3) ser mayor de 18 años. Las participantes no recibieron ningún tipo de incentivo por su participación. La aplicación piloto de la escala se realizó durante el mes de mayo de 2019 en la región de Valparaíso.

\section{Instrumento}

La escala de VO fue creada a partir del denominado "test de violencia obstétrica" elaborado por la asociación El parto es nuestro (2014), que incluye las situaciones más recurrentes de falta de respeto y abuso en la atención obstétrica. Dicho instrumento fue modificado en su redacción (de modo de adaptarlo a una escala en formato de respuesta graduada) y ampliado para atender situaciones no contempladas en la propuesta citada, particularmente aquellas situaciones referidas a la exposición a la mirada de otras personas que no son parte del equipo médico y referidas a la posibilidad de utilizar algún dispositivo o realizar algún procedimiento de control de natalidad con posterioridad al parto (DIU, ligadura de trompas, etcétera). Del mismo modo, algunas opciones de dicho test fueron desagregadas de modo que no contuvieran más de una idea central por ítem. Dicho test no constituye una escala de medida, sino que simplemente representa una serie categorías de violencia que pueden responderse de forma dicotómica ( $S i$ o No) o que son ejemplificadas de manera de ayudar a las mujeres a reconocer si han sufrido algún tipo de violencia. De allí que la variabilidad de respuestas que permitan incorporar sea sumamente baja, dado que hay cuestiones que afectan a las personas en grados diferentes (lo que el instrumento original no permitiría captar). Su intención no es generar un instrumento útil para la investigación, sino para su uso en el contexto clínico y de información. Dado los usos para los que fue creado, dicho instrumento no posee indicaciones de validez o confiabilidad, lo que limita su uso en el ámbito clínico y de investigación, esto es, no puede dar cuenta de qué es lo que realmente mide y qué tan bien lo hace. A pesar de ello, se trata de una herramienta con mucho potencial desarrollada desde el activismo y que supone recoger, de muy buena manera, ciertas prácticas que pueden categorizarse como VO. De allí nuestro interés en sistematizar y mejorar dicho instrumento, partiendo por comprobar si su estruc- 
tura se ajusta al modelo particular hipotetizado y si dicho modelo es utilizable a lo largo de diferentes muestras, lo que permitiría realizar comparaciones válidas entre ellas.

La escala final consta de 14 ítems en formato Likert con cinco opciones de respuesta (que van desde 1 = No describe para nada lo que me ocurrió, hasta 5 = Definitivamente esto me ocurrió; ver Anexo). La redacción de los ítems fue revisada por un grupo de expertas del área de la salud de las mujeres. Dicho panel contribuyó a mejorar la redacción de los reactivos y hacer que estos resultaran representativos de las situaciones más corrientes reportadas en estudios previos sobre problemas de salud ginecológica y del ámbito obstétrico. El panel fue conformado por diversas mujeres que trabajan en colectivos sobre derechos reproductivos y VO (entre las que había matronas, enfermeras, abogadas y activistas) que revisaron el instrumento y realizaron los comentarios pertinentes.

Además de la aplicación de la escala de VO, se consultó a las participantes por la realización de una serie de prácticas (11 en total), ejecutadas por miembros del equipo médico durante su parto, que en este contexto pueden considerarse rutinarias y que, de existir relación entre su ejecución y la VO reportada, servirían como indicadores de validez de la escala. Dichas prácticas refieren al rasurado de genitales, la prohibición de tomar líquidos o comer durante el trabajo de parto, las indicaciones de permanecer acostada durante el trabajo de parto, el enema (lavado intestinal), el uso de medicamentos para apurar el parto, la rotura artificial de membrana, la compresión del abdomen al momento de los pujos (maniobra de Kristeller), el legrado o raspaje de útero sin anestesia, la cesárea, los tactos vaginales reiterados y la episiotomía. El formato de respuesta era de carácter dicotómico (Sí o No) y apuntaba a detectar presencia o ausencia del procedimiento.

\section{Análisis de datos}

Se usaron los programas SPSS 20.0 y AMOS 22 para todos los análisis. Se realizó un análisis descriptivo (media, desviación estándar, simetría y curtosis) en todas las variables para observar la forma en que se distribuyen los datos, dado que la mayor parte de las pruebas estadísticas se basa en el supuesto de normalidad de la distribución. Se calculó el coeficiente de confiabilidad de la escala (alfa de Cronbach y omega). El coeficiente alfa es el más utilizado en estudios psicométricos y constituye una medida de consistencia interna de la escala, pero desde hace un tiempo el coeficiente omega ha demostrado ser mejor indicador para datos de tipo ordinal (McDonald, 1999), toda vez que estaría menos afectado por el número de ítems y de la variabilidad producida por la reducción de las alternativa de respuestas (Ventura-León y Caycho-Rodríguez, 2017), ya que se calcularía sobre la base de las cargas factoriales, lo que haría más estables los cálculos. Los análisis de fiabilidad permitirán ver cuán preciso es el instrumento de medida para captar el constructo VO. El modelo unifactorial se probó por medio de un análisis factorial de tipo confirmatorio (método de máxima verosimilitud). Los análisis factoriales confirmatorios multigrupo, mediante un procedimiento por pasos, establecieron la invariancia de la escala en muestras constituidas por mujeres que tuvieron sus partos en el sistema público de salud o en el sistema privado. Lo que se pretende mostrar en este nivel es que la estructura de la variable VO es unidimensional y que esa dimensión estaría adecuadamente cubierta por los ítems de la escala. Las pruebas de invarianza tienen como objetivo asegurar que el constructo que se evalúa (en este caso la VO) tiene el mismo significado para las mujeres que realizan su atención en el sistema privado y público de salud, lo cual permitiría posteriormente su aplicación en dichos ámbitos y la comparación entre grupos sin riesgo de que se estén comparando cuestiones que para cada grupo tienen un significado diferente. Este procedimiento comenzó estimando el modelo de línea de base ( $\sin$ restricciones) $\mathrm{y}$, en modelos posteriores, se 
agregaron progresivamente restricciones mientras se mantenían constantes las restricciones anteriores. Las restricciones se impusieron en las cargas factoriales, las varianzas y las covarianzas. Los modelos anidados se compararon en cada paso mediante el examen de la prueba de diferencias de chi-cuadrado $\left(\chi^{2}\right)$ y el cambio en los valores del Comparative Fit Index (CFI), así como los otros índices de ajuste. La comparación de modelos y el establecimiento de la invarianza métrica se realizó considerando dos criterios: si las estadísticas de la diferencia de chi-cuadrado no revelan diferencias significativas entre el modelo de línea de base y cada modelo subsiguiente, podemos asumir la invariabilidad del modelo (Byrne, 2001). Por otro lado, los cambios en el valor del CFI deben ser inferiores a .01 para establecer la invariancia (Cheung y Rensvold, 2002). Finalmente se realizaron pruebas de asociación (chi-cuadrado) entre las 11 prácticas que pudieron haber sido realizadas por integrantes del equipo médico y el reporte de VO por parte de las participantes. Estos análisis servirán como indicación adicional de la validez del constructo.

\section{Resultados}

La Tabla 1 muestra los estadísticos descriptivos para cada uno de los ítems de la aplicación piloto (medias, desviaciones estándar, índices de asimetría y curtosis). Todas las medias se encuentran por debajo del punto medio de la escala y muestran niveles de dispersión relativamente similares. Un análisis de los ítems de la escala no indica presencia de casos atípicos o extremos en las respuestas a los ítems. La distribución de la mayoría de los elementos muestra una asimetría positiva, lo que indica que los datos se agrupan hacia el área izquierda de la distribución, indicando un menor reporte de situaciones de violencia. El nivel de curtosis revela que las respuestas acumuladas en las colas de distribución son ligeramente más altas que en una curva normal. La distribución de respuestas en la escala total muestra cierta asimetría negativa y una distribución leptocúrtica, lo que mostraría que la distribución no se asemeja a una curva normal.

Tabla 1

Estadísticos descriptivos.

\begin{tabular}{c|c|c|c|c} 
Ítem & $\mathbf{M}$ & $\mathbf{S D}$ & Asimetría & Curtosis \\
\hline 01 & 1.67 & 1.39 & 1.76 & 1.32 \\
02 & 1.55 & 1.27 & 2.69 & 9.45 \\
03 & 1.79 & 1.43 & 1.48 & .48 \\
04 & 2.07 & 1.65 & 1.02 & -.79 \\
05 & 2.11 & 1.63 & .99 & -.81 \\
06 & 1.80 & 1.46 & 1.48 & .46 \\
07 & 2.05 & 1.63 & 1.08 & -.64 \\
08 & 1.98 & 1.59 & 1.18 & -.41 \\
09 & 1.97 & 1.62 & 1.19 & -.44 \\
10 & 1.73 & 1.45 & 1.63 & .85 \\
11 & 1.41 & 1.42 & 8.07 & 9.87
\end{tabular}




\begin{tabular}{c|c|c|c|c} 
Ítem & $\mathbf{M}$ & SD & Asimetría & Curtosis \\
\hline 12 & 1.95 & 1.61 & 1.53 & 2.00 \\
13 & 1.32 & 0.98 & 3.02 & 7.82 \\
14 & 1.90 & 1.54 & 1.30 & -.07 \\
Escala VO & 1.80 & .93 & 1.27 & .95
\end{tabular}

\section{Confiabilidad}

La escala de VO muestra un coeficiente de confiabilidad de .83 (alfa de Cronbach) para el conjunto de los ítems. Las correlaciones de los ítems con el total fluctúan en un rango entre .29 y .71. Del mismo modo, la fiabilidad obtenida con base en las cargas factoriales (omega) fue de .88. Las cargas factoriales fluctuaron entre .26 y .78 .

\section{Análisis factorial confirmatorio}

Se puso a prueba un modelo unifactorial referido a VO. Los parámetros del modelo fueron estimados utilizando el método de máxima verosimilitud. Se obtuvieron los índices de ajuste del modelo y las estimaciones de las cargas factoriales para cada ítem. Se utilizaron varios índices para estimar la bondad de ajuste del modelo: chi-cuadrado, índice de ajuste comparativo (CFI), índice de ajuste incremental (IFI), coeficiente de Tucker-Lewis (TLI) y error de aproximación (RMSEA). Dichos índices de bondad de ajuste permiten evaluar la exactitud de los datos del modelo para determinar si este es correcto, mediante la evaluación del ajuste global del modelo. Se espera que el chi-cuadrado no sea significativo (aunque este indicador no debe usarse para descartar un modelo debido a su sensibilidad al tamaño de la muestra). TLI, IFI y CFI deberían ser los más cercanos a 1 , aunque siempre se espera que sean más altos que .90 (Bentler y Dudgeon, 1996; Hu y Bentler, 1995). El valor RSMEA debe ser inferior al valor crítico de .08 (Browne y Cudeck, 1993).

Los datos obtenidos muestran un buen ajuste con el modelo teórico que afirma la no unidimensionalidad del constructo VO (ver Tabla 2) porque, a pesar de que el valor de chi-cuadrado es significativo $\left(\chi_{(74)}^{2}=194.71\right.$, $p<.001)$, los valores de CFI (.94), IFI (.94) y TLI (.93) muestran claramente un buen ajuste de datos (el modelo hipotético debe rechazarse porque lo que se contrasta es la hipótesis nula de ajuste perfecto entre datos empíricos y teóricos). Finalmente, el valor de RMSEA fue .067 (IC del $95 \%[.055, .078]$ ), lo que indica un buen ajuste general del modelo. Las cargas factoriales de todos los elementos oscilan entre .26 y .78 .

Se realizaron dos análisis factoriales confirmatorios separados para las mujeres que habían tenido sus partos en instituciones públicas o privadas (ver Tabla 2). El modelo unifactorial proporcionó un buen ajuste para ambos: salud pública: $\chi_{(74)}^{2}=146.83, p<.001, \mathrm{CFI}=.93, \mathrm{IFI}=$ $.93, \mathrm{TLI}=.91$ y $\mathrm{RMSEA}=.072$; salud privada: $\chi_{(74)}^{2}=168.56, p<.001, \mathrm{CFI}=.92, \mathrm{IFI}=.92$, $\mathrm{TLI}=.89$ y RMSEA $=.091$. La realización de análisis separados supone que la VO se produce indistintamente del tipo de centro en el que las mujeres atienden sus partos y que, por lo mismo, la escala debe servir para captar el significado de dicha violencia en instituciones públicas o privadas. Los índices de ajuste mostraron valores adecuados para los dos grupos, lo que sugiere que el modelo de un factor general puesto a prueba resulta pertinente para detectar $\mathrm{VO}$ en mujeres que se atienden en ambos tipos de instituciones, aunque si atendemos a los resultados referidos al error cuadrático medio de aproximación (RMSEA), estos son ligeramente mejores en el caso del grupo atendido en la salud pública, lo cual podría explicarse por el bajo tamaño muestral y el elevado número de parámetros a estimar. 
Tabla 2

Análisis factorial confirmatorio y pruebas de invarianza.

\begin{tabular}{c|c|c|c|c|c} 
Modelos & $\chi^{2}(d f)$ & CFI & IFI & TLI & $\begin{array}{c}\text { RSMEA }(\text { IC95 } \\
\mathbf{\%})\end{array}$ \\
\hline $\begin{array}{c}\text { 1. Modelo de medida } \\
\text { Modelo de un factor }\end{array}$ & $194.71(74)$ & .94 & .94 & .93 & $.067(.055-.078)$ \\
\hline $\begin{array}{c}\text { 2. Análisis de muestras } \\
\text { Salud Pública }\end{array}$ & $146.83(74)$ & .93 & .93 & .91 & $.072(.055-.089)$ \\
Salud Privada & $168.56(74)$ & .92 & .92 & .89 & $.091(.073-.109)$ \\
\hline $\begin{array}{c}\text { 3. Análisis multigrupo } \\
\text { Modelo sin restricciones } \\
\text { Restricción de cargas facto- } \\
\text { riales (FL) }\end{array}$ & & & & & \\
$\begin{array}{c}\text { FL + Restricción de } \\
\text { varianzas (V) }\end{array}$ & & .921 & & & $.058(.049-.066)$ \\
$\begin{array}{c}\text { FL + V + Restricción de } \\
\text { covarianzas }\end{array}$ & & .911 & & & $.059(.050-.067)$ \\
& & .877 & & & $.058(.050-.057)$ \\
\end{tabular}

\section{Pruebas de la invarianza a través de muestras}

Los resultados de la varianza multigrupo que se presentan en la Tabla 2 indican que el modelo de línea de base (sin restricciones) mostró que el modelo hipotético de la estructura de la escala de VO encaja bien en los dos grupos. Al comparar los modelos subsiguientes con restricciones adicionales al modelo de línea de base (en el Modelo A, se impusieron restricciones a las cargas factoriales; en el Modelo B, se impusieron restricciones a las cargas y varianzas de los factores; y en el Modelo C, se impusieron restricciones a las cargas factoriales, varianzas y covarianzas), los cambios en los valores de CFI y RMSEA fueron menores a .01 (salvo para el caso de la invarianza fuerte y sólo con las variaciones de CFI) y permiten establecer la invariancia de la escala. Dicha invarianza vendría a significar que existe evidencia de que el constructo evaluado (VO) posee el mismo significado para las mujeres pertenecientes a los dos grupos comparados (prestadores públicas o privados de servicios de salud).

\section{Prevalencia de VO y su relación con otros procedimientos médicos realizados durante el parto}

Del total de las mujeres que participaron del estudio un $58.9 \%$ declara haber vivido alguna situación de violencia durante la atención de su parto. La Tabla 3 muestra las frecuencias acumuladas para las respuestas a cada pregunta del cuestionario. Como se puede apreciar, una proporción elevada de mujeres (entre un $20 \%$ y un $25 \%$ ) manifiesta haber sido criticada por el personal médico por realizar expresiones emocionales o de dolor (25.6\%), no pudiendo manifestar sus inquietudes o temores dado que no le respondían o lo hacían de mala manera $(24.9 \%)$. Al mismo tiempo, los reportes de la obligatoriedad de permanecer acostada en cama $(22.4 \%)$ y en posición boca arriba (23 
$\%$ ), a pesar del dolor que sentían, hablan de la imposición sistemática de este tipo de prácticas. El impedimento de estar acompañadas es otra de las prácticas que implica la falta de respeto o abuso que más se repiten entre las mujeres de la muestra (23.2\%). Entre las prácticas menos mencionadas se encuentra el hecho de hacer sentir a las mujeres que no habían colaborado o estado a la altura de lo que se esperaba de ellas $(6.6 \%)$ o la negativa a utilizar dispositivos de control de natalidad como DIU o ligadura de trompas (6.9\%).

Tabla 3

Frecuencias de respuesta para cada pregunta.

\begin{tabular}{|c|c|c|c|c|c|}
\hline Ítem & $\begin{array}{l}\text { "No describe para } \\
\text { nada lo que me } \\
\text { ocurrió" }(\%)\end{array}$ & $\begin{array}{c}\text { "No estoy segura, } \\
\text { pero creo/siento } \\
\text { que no me } \\
\text { ocurrió" }(\%)\end{array}$ & $\begin{array}{l}\text { "No estoy } \\
\text { segura" } \\
(\%)\end{array}$ & $\begin{array}{l}\text { “No estoy } \\
\text { segura } \\
\text { pero creo/ } \\
\text { siento que sí } \\
\text { ocurrió" (\%) }\end{array}$ & $\begin{array}{l}\text { "Definitiva- } \\
\text { mente esto me } \\
\text { ocurrió" (\%) }\end{array}$ \\
\hline 01 & 78.7 & 3.6 & 1.6 & 4.1 & 12.0 \\
\hline 02 & 80.3 & 3.8 & 4.1 & 5.5 & 6.3 \\
\hline 03 & 73.2 & 4.7 & 4.1 & 6.3 & 11.8 \\
\hline 04 & 67.8 & 3.0 & 3.6 & 5.7 & 19.9 \\
\hline 05 & 63.9 & 6.0 & 5.2 & 5.2 & 19.7 \\
\hline 06 & 73.0 & 5.7 & 3.0 & 4.6 & 13.7 \\
\hline 07 & 67.1 & 3.8 & 6.0 & 3.0 & 20.0 \\
\hline 08 & 69.2 & 4.4 & 4.1 & 4.1 & 18.3 \\
\hline 09 & 71.4 & 2.7 & 2.7 & 4.1 & 19.1 \\
\hline 10 & 77.9 & 2.7 & 1.9 & 3.5 & 13.9 \\
\hline 11 & 85.4 & 4.7 & 3.3 & 1.9 & 4.7 \\
\hline 12 & 70.4 & 3.9 & 4.7 & 5.0 & 16.1 \\
\hline 13 & 88.7 & 1.9 & 2.5 & 2.2 & 4.7 \\
\hline 14 & 71.0 & 4.1 & 5.0 & 3.3 & 16.6 \\
\hline
\end{tabular}

En cuanto a la realización de otros procedimientos rutinarios asociados al trabajo de parto, hay una alta incidencia de prácticas rutinarias y que exceden con mucho los porcentajes anteriores: rasurado (70.6 \%), realización de enema (51.2\%), prohibición de tomar líquidos o comer $(65.2 \%)$, indicación de permanecer acostada durante el trabajo de parto $(24.5 \%)$, uso de medicamentos para apurar el parto (46.6\%), rotura artificial de membrana (42\%), maniobra de Kristeller o compresión del útero en el momento de los pujos $(42.5 \%)$, tactos vaginales reiterados $\mathrm{y}$ realizados por diferentes integrantes del equipo médico $(24.3 \%)$ o legrado de útero sin anestesia $(4.5 \%)$. Entre quienes no se realizaron cesárea (45.9\%), a un $74.11 \%$ se le realizó una episiotomía; esto es, se les realizó 
un corte en la zona del perineo con la finalidad de agrandar el canal del parto (lo que nos habla de lo rutinario de este tipo de procedimientos). En todos estos procedimientos existe una alta proporción que resulta innecesario y que, de ser rotulados como formas de ejercicio de violencia, aumentarían con mucho el porcentaje de mujeres que, conscientes o no de ello, han vivido formas de violencia durante su trabajo de parto.

Al realizar pruebas de asociación $\left(\chi^{2}\right)$ entre el reporte de estas prácticas y el afirmar haber vivido VO (aquellas mujeres que, en al menos una de las preguntas de la escala de VO, estaban completamente seguras de la ocurrencia del hecho referido por el ítem) se observa una asociación significativa con la prohibición de consumir líquidos o alimentos durante el parto $\left(\chi^{2}=20.385, p<.001\right.$, phi $=.24)$, la indicación de permanecer acostada durante el trabajo de parto $\left(\chi^{2}=26.54, p<\right.$ .001 , phi $=.27)$, la administración de medicamentos para apurar el parto $\left(\chi^{2}=12.737, p<\right.$ $.001, \mathrm{phi}=.19)$, el tacto vaginal reiterado $\left(\chi^{2}\right.$ $=21.647, p<.001$, phi $=.24)$ y la compresión de abdomen durante el parto $\left(\chi^{2}=6.607\right.$, $p=.010, \mathrm{phi}=.14)$. La asociación entre estas prácticas y el reporte de $\mathrm{VO}$ puede ser indicador de la validez de la escala de VO.

Finalmente, resulta llamativo que, entre las mujeres que no reportan haber vivido VO, sí se reporta la presencia de algunas de las prácticas antes señaladas: rasurado de genitales $(66.2 \%)$, enema (45.5\%), prohibición de consumir alimentos o agua (51.7\%), indicación de permanecer acostada durante el trabajo de parto (24.5\%), inducción del parto mediante uso de medicamentos $(36.1$ $\%)$, tactos vaginales reiterados $(24.3 \%)$, compresión del abdomen (34.5\%), episiotomía (33.6\%), cesárea (54.1\%) y raspaje de útero sin anestesia (2\%). Los resultados anteriores indican la normalización de las prácticas violentas y su realización rutinaria en el marco de la atención de los partos en servicios de salud públicos y privados, lo que podría hacer subestimar su prevalencia debido a su subreporte, dada su normalización y legitimación, que hace que una alta proporción de las mujeres no las reconozcan como formas de violencia en el marco de su atención de salud.

\section{Discusión}

El propósito de este artículo fue adaptar y validar una escala para detectar VO. En términos generales, la escala muestra buenos niveles de confiabilidad y validez. Se trata de una escala suficientemente breve como para utilizarla en el ámbito de la investigación, pero que logra captar adecuadamente la dimensionalidad del constructo. Tal como se esperaba, el modelo de un factor general obtiene buenos niveles de ajuste. Del mismo modo, el modelo muestra buen ajuste a través de distintas muestras (diferentes servicios de salud en los que se atendió su parto), sugiriendo que el modelo puesto a prueba es una herramienta aplicable a diferentes contextos de atención.

El instrumento adaptado, y del cual se han entregado algunas indicaciones de validez, debe seguir acumulando evidencia que autorice su uso en contextos de investigación diversos, así como en el campo clínico, y puede asumirse como una propuesta inicial que debe acumular mayor evidencia en vistas a constituirse en una herramienta útil en la detección de diversas formas de VO. Del mismo modo, futuros estudios deben superar ciertas limitaciones presentes en este artículo. La primera es el uso de una muestra de carácter no probabilístico, lo que dificulta la generalización de los resultados a la población general. Por otra parte, se espera comenzar estudios que permitan poner a prueba esta escala en otros colectivos que deben utilizar servicios públicos o privados para su atención obstétrica, tales como las personas transgénero e inmigrantes y que son quienes, de forma más evidente, sufren el rechazo de un modelo médico basado en la segmentación y jerarquización.

En conclusión, los resultados fueron consistentes con las hipótesis y muestran que 
la escala de VO puede transformarse en un instrumento robusto (confiable y válido) para detectar esta particular forma de violencia hacia las mujeres. Estos instrumentos pueden permitir recoger adecuadamente una experiencia hasta ahora negada, o cuando menos naturalizada, de modo de visibilizar un fenómeno con secuelas sumamente negativas para la salud física (presencia de dolores de carácter permanente en la zona de la episiotomía, roturas producto de la compresión del abdomen al momento de los pujos, fístulas, incontinencia urinaria, etcétera) y de graves consecuencias psíquicas en quienes desean realizar un parto dentro de las instituciones de salud. De allí que cada día cobren más fuerza aquellas acciones que llevan a las personas a realizar su parto de forma no institucionalizada, pero más respetada, acompañada y por sobre todo, en ausencia de abuso.

\section{Referencias bibliográficas}

Almonte, G. (2016). Violencia ginecológica y obstétrica. La medicalización del cuerpofemenino. Revista Décsir, 2, 1-10.

Arguedas, G. (2014). La violencia obstétrica: propuesta conceptual a partir de la experiencia costarricense. Cuadernos intercambio sobre Centroamérica y el Caribe, 11(1), 145-169. https://doi.org/10.15517/c.a.v11i1.14238

Asefa, A. y Bekele, D. (2015). Status of respectful and non-abusive care during facility-based childbirth in a hospital and health centers in Addis Ababa, Ethiopia. Reproductive Health, 12(33), 1-9.

Belli, L. (2013). La violencia obstétrica: otra forma de violación a los derechos humanos. Revista Redbioética / UNESCO, 1(7), 25-34.

Bellón, S. (2015). La violencia obstétrica desde los aportes de la crítica feminista y la biopolítica. Dilemata, 18, 93-11.

Benjamin, J. (2012). El Tercero. Reconocimiento. Clínica e Investigación Relacional, 6(1), 169-179.

Bentler, P. M. y Dudgeon, P. (1996) Covariance structure analysis: Statistical practice, theory and directions. Annual Review of Psychology, 47, 563-592. https://doi.org/10.1146/annurev .psych.47.1.563

Bowser, D. y Hill, K. (2010). Exploring evidence for disrespect and abuse in facility-basedchildbirth: report of a landscape analysis. USAID-TRAction Project: Harvard School of Public Health University Research Co, LLC: $1-57$.

Browne, M. W. y Cudeck, R. (1993). Alternative ways of assessing model fit. En K. A. Bollen y J. S. Long (Eds.), Testing structural equation models (pp. 136-162). Newbury Park, CA: Sage.

Brüggemann, J. y Swahnberg, K (2014). Staff silence about abuse in health care: An exploratory study at a Swedish women's clinic. Clinical Ethics, 9(2-3), 71-76. https://doi. org/10.1177/1477750914546760

Byrne, B. (2001). Structural equation modeling with AMOS. Basic concepts, applications, and programing (2nd ed.). New Jersey, NJ: Lawrence Erlbaum Associates.

Calafell, N. (2015). La violencia obstétrica y sus modelos de mundo. Cuestiones de Género de la Igualdad y la Diferencia, 10, 331-354.

Cheung, G. y Rensvold, R. (2012). Evaluating goodness-of-fit indexes for testing measurement invariance. Structural Equation Modeling, 9, 233-255. https://doi.org/10.1207/ S15328007SEM0902_5

Cohen, S. (2016). Making Loud Bodies "Feminine": A Feminist-Phenomenological Analysis of Obstetric Violence. Human Studies, 39, 231-247. https://doi.org/10.1007/s10746-0159369-X

Contreras, N. y Fernández, C. (2015). El parto medicalizado en Chile. Saberes, capitalismo y patriarcado Chile. Santiago: Pensamiento y Batalla.

De Marco, M., Sheryl, T. y Zhao, W. (2008). Perceived Discrimination During Prenatal Care, Labor, and Delivery: An examination of data from the Oregon pregnancy risk assessment monitoring system, 1998-1999, 2000, and 2001. American Journal of Public Health, 90(10), 1818-1822. 
D’Oliveira A. F., Diniz S. G., y Schraiber L. B. (2002). Violence against women in health-care institutions: an emerging problem. Lancet, 359(9318), 1681-1685.

El parto es nuestro (2014). Test de violencia obstétrica. https://www.elpartoesnuestro.es/ blog/2014/08/18/test-de-violencia-obstetrica.

Faneite, J., Feo, A. y Toro, J. (2012). Grado de conocimiento de violencia obstétrica por el personal de salud. Revista de Obstetricia y Ginecología de Venezuela, 72(1), 4-12.

Fernández-Guillén, F. (2015). ¿Qué es la violencia obstétrica? Algunos aspectos sociales, éticos y jurídicos. Dilemata, 18, 113 - 128.

Gonçalves da Silva, M., Carreira, M., Pinheiro, L., Carcaman, R. y Kakuda, A. (2014). Obstetric violence according obstetric nurses. Revista Rene, 15(4), 720-728. https://doi. org/10.15253/2175-6783.2014000400020

Hernández-Garre, J. M. y Echevarría-Pérez, P. (2016). Sociología del parto. Conflictos asistenciales en el marco del paradigma tecnocrático. Revista Internacional de Sociología, 74(1), e025. https://doi.org/10.3989/ ris.2016.74.1.025

Hu, L. T. y Bentler, P. M.. (1995). Evaluating model fit. En R. H. Hoyle (Ed.), Structural equation modeling: Concepts, issues, and applications (pp. 76-99). Thousand Oaks, CA: Sage.

Infante, G. y Leiva, G. (2016). Violencia Obstétrica. En C. Dides y C. Fernández (Eds.), Primer Informe. Salud Sexual, Salud Reproductiva y Derechos Humanos en Chile. MilesChile.

Instituto de Género, Derecho y Desarrollo INSGENAR. (2003). Con todo al aire 1. Reporte de derechos humanos sobre atención en salud reproductiva en hospitales públicos. Rosario, Argentina. https://insgenar.files. wordpress.com/2012/04/con-todo-al-aire.pdf

Instituto Nacional de Derechos Humanos - INDH. (2016). Informe Anual. Situación de los Derechos Humanos en Chile. Santiago.

Kruk M., Kujawski, S., Mbaruku, G., Ramsey, K., Moyo, W. y Freedman, L. (2014). Disrespectful and abusive treatment during facility delivery in Tanzania: a facility and commu- nity survey. Health Policy y Planning, 33(1), 26-33. https://doi.org/10.1093/heapol/czu079.

Lukasse M., Schroll, A. M., Karro, H., Schei, B., Steingrimsdottir, T., Van Paris, A. S., Ryding, E .L. y Tabor, A. (2015). Prevalence of experienced abuse in healthcare and associated obstetric characteristics in six European countries. Acta Obstetricia et Gynecologica Scandinavica, 94(5), 508-517. https://doi. org/10.1111/aogs.12593.

Majety, C. y Bejugam, S. (2017). Challenges and baries faced by women in accesing justice against obstetric violence. International Journal of Reproduction Contraception Obstetrics and Gynecology, 5(9), 2899-2903. https:// doi.org/10.18203/2320-1770.ijrcog20162968

McDonald, R. P. (1999). Test theory: A unified treatment. Mahwah: Lawrence Erlbaum Associates, Inc.

Nacer en libertad (2015). Memorias de parto. Relatos de mujeres para renacer juntas. Santiago: Quimantú.

Organización Mundial de la Salud - OMS. (2014). Prevención y erradicación de la falta de respeto y maltrato durante la atención del parto en centros de salud. https://apps. who.int/iris/bitstream/handle/10665/134590/ WHO_RHR_14.23_spa.pdf;jsessionid=6BF04FAA 214632AE2E5E26AB26550E01?sequence $=1$

Observatorio de Violencia Obstétrica - OVO. (2018). Resultados Primera Encuesta sobre el Nacimiento en Chile. Santiago, Chile. https:// ovochile.cl/descarga-resultados-primera-encuesta-sobre-el-nacimiento-en-chile/

Respectful Maternity Care Advisory Council, White Ribbon Alliance for Safe Motherhood (2011). Respectful maternity care: the universal rights of childbearing women. Washington, DC: WRA. www.whiteribbonalliance.org/ index.cfm/the-issues/respectful-maternity-care

Red chilena contra la violencia hacia las mujeres. (2015). El continuo de violencia hacia las mujeres y la construcción de nuevos imaginarios. Santiago, Chile.

Sadler, M., Santos, M., Ruiz-Berdum, D., Leiva, G., Skoko, E., Guillen, P. y Clausen, 
J. (2016). Moving beyond disrespect and abuse: addressing the structural dimensions of obstetric violence. Reproductive Health Matters, 24(47), 47-55. https://doi. org/10.1016/j.rhm.2016.04.002

Salinero, S. (2017). El parto en casa una respuesta a la violencia obstétrica e institucional. El Desconcierto. http://www.eldesconcierto. cl/2017/12/11/el-parto-en-casa-una-respuesta-a-la-violencia-obstetrica-e-institucional/.

Salinero, S. (2021). Fue un terremoto: violencia obstétrica y relatos de mujeres en Chile. Debate Feminista, 62 (en prensa).

Savage, V. y Castro, A. (2017). Measuring mistreatment of women during childbirth: a review of terminology and methodological approaches. Reproductive Health, 14 (138). https://doi.org/10.1186/s12978-017-0403-5.

Sena, L. M. (2016). Ameaçada e sem voz, como num campo de concentração. A medicalizaçao do parto como porta e palco para a violência obstétrica (Tesis de Doctorado). Programa de Postgrado en Salud Colectiva, Centro de Ciencias de la Salud, Universidad General de Santa Catarina, Florianópolis, Brasil.

Schröll, A. M. y Midtgaard, J. (2013). Encountering abuse in health care; lifetime experiences in postnatal women - a qualitative study. Pregnancy and Childbirth, 13(74). https://doi. org/10.1186/1471-2393-13-74
Swahnberg, K., Thapar-Björkert, S. y Bertero, C. (2007). Nullified: women's perceptions of being abused in health care. Journal of Psychosomatic Obstetrics \& Gynaecology, 28, 161-167. https://doi.org/10.1080/01674820601143211.

Swahnberg, K. y Wijma, B. (2003). The NorVold Abuse Questionnaire (NorAQ). Validation of new measures of emotional, physical, and sexual abuse, and abuse in the health care system among women. European Journal of Public Health, 13, 361-366. https://doi. org/10.1093/eurpub/13.4.361.

Tamayo, J., Restrepo, C., Gil, L. y González, A. M. (2015). Violencia Obstétrica y Aborto. Aportes para el debate en Colombia. Grupo Médico por el derecho a decidir.

Terán P, Castellanos, C., González, M. y Ramos, D. (2013). Violencia obstétrica: percepción de las usuarias. Revista de Obstetricia y Ginecología de Venezuela, 73(3), 171-180.

Valdez-Santiago, R. et al. (2015). Nueva evidencia a un viejo problema: el abuso de las mujeres en las salas de parto. Revista CONAMED, 18(1), 14-20.

Ventura-León, J. L. y Caycho-Rodríguez, T. (2017). El coeficiente Omega: un método alternativo para la estimación de la confiabilidad. Revista Latinoamericana de Ciencias Sociales, Niñez y Juventud, 15(1), 625-627.

Recibido: 19 de noviembre de 2019 Aceptado: 9 de marzo de 2021 


\section{Anexo}

\section{Escala de Violencia Obstétrica}

1. El personal de salud hizo comentarios irónicos, descalificadores o en tono de chiste acerca de tu comportamiento.

2. Te trataron con sobrenombres o diminutivos (p.e. "mamita", "gordita", etc.) o como si fueras incapaz de comprender los procesos por los cuales estabas atravesando.

3. Te sentiste infantilizada o anulada por el equipo médico como si fueras incapaz de tomar decisiones sobre lo que te ocurría antes, durante o después del parto.

4. Fuiste criticada de algún modo por expresar de algún modo tus emociones (llorar, gritar de dolor, etc.) durante trabajo de parto y/o el parto.

5. Te fue difícil o imposible preguntar o manifestar tus miedos o inquietudes porque no te respondían o lo hacían de mala manera.

6. Te realizaron procedimientos médicos sin pedirte consentimiento o sin explicarte por qué eran necesarios.

7. En el momento del parto, te obligaron a permanecer acostada boca arriba aunque manifestaras tu incomodidad en esa posición.

8. Fuiste obligada a quedarte en cama impidiéndote caminar o buscar posiciones según tus necesidades.

9. Se te impidió estar acompañada por alguien de tu confianza.

10. Se te impidió el contacto inmediato con tu hija/o recién nacida/o antes de que se lo llevara la/ el neonatólogo para control (acariciarlo, tenerlo en brazos, etc.).

11. Después del parto, te hicieron sentir que no habías estado a la altura de lo que se esperaba de ti (que no habías «colaborado»).

12. La experiencia de la atención en el parto te hizo sentir vulnerable, culpable o insegura en algún sentido.

13. Después del parto se te negó la posibilidad de utilizar algún dispositivo o realizar algún procedimiento de control de natalidad (DIU, ligadura de trompas, etc.).

14. Durante el trabajo del parto o con posterioridad a este te sentiste expuesta a la mirada de otras personas desconocidas para ti (exposición a extraños). 